\title{
A Biomechanical Study of the Medial Row Tightening on Stability of Single Stitch Suture-Bridge Construct
}

Sharon Abihssira ( $\nabla$ sharon@abihssira.net)

Clinique Maussins-Nollet https://orcid.org/0000-0001-8782-9396

Pierre-Alban BOUCHE

Clinique Maussins-Nollet

Claire CAMMAS

Clinique Maussins-Nollet

Clément THIRACHE

Clinique Maussins-Nollet

Geoffroy NOURISSAT

Clinique Maussins-Nollet

\section{Research Article}

Keywords: suture-bridge, biomechanics, limb, knot, sutures, tapes, rotator cuff

Posted Date: January 17th, 2022

DOI: https://doi.org/10.21203/rs.3.rs-1241917/v1

License: (c) (i) This work is licensed under a Creative Commons Attribution 4.0 International License. Read Full License 


\section{Abstract}

Purpose: To define the more stable knot tightening of a suture-bridge when a single limb is preserved.

\section{Methods:}

Five different sutures were tested: No. 2 Ethibond (Ethicon), Hi-Fi (ConMed Linvatec), Sutblue (SBM), SingleFlat (SBM), Hi-Fi Ribbon (ConMed Linvatec).

A Surgeon's Knot was tied around a 30-mm circumference device, 6 times for each experiment. A single limb was kept to analyze failure modes of the knot.

First step was to analyze which of pulling or sliding suture of the construct must be kept preventing failure of the knot.

The cutting distance from the knot was evaluated at $1 \mathrm{~mm}$ and $4 \mathrm{~mm}$ with the suture loops pretensioned to $10 \mathrm{~N}$ and fixed to a second row after a $50 \mathrm{~N}$ tension load.

The more stable construct was found: a single-pull load to100 $\mathrm{N}$ and cyclic load (to $50 \mathrm{~N}$ for 30 cycles) experiments were conducted to evaluate the impact of cycling on knot loosening.

Results: The more stable construct was obtained when the non-post limb was tensioned, and the postlimb was cut at $4 \mathrm{~mm}(p<0.01)$. Loop circumference increased after each experiment for all tested sutures, independently of the preserved limb and the cutting distance. Elongation was significant for all tested sutures in all groups. Knot failure mostly occurred by slippage; only with tapes.

Conclusions: a suture bridge construct with the non-post limb preserved and the post-limb cut at a $4 \mathrm{~mm}$ distance from the knot provides with the best security. Sutures are safer than tapes in suture-bridge.

\section{Introduction}

Rotator cuff tears are mainly treated with arthroscopic methods after an initial medical management. Among them, suture-bridge or transosseous equivalent is a validated technique. It appears to be associated with a primary higher tendon healing rate and a reduced rate of retear compared to single-row repair [1, 2]. Different types of medial row configurations have been proposed. Suture bridge is different from speed bridge by the fact that the first row is tightened with knots, allowing compression and independent fixation at the medial row [3]. Double-mastress suture bridge combines a high fixation strength without mechanical failure [4]. This construct allows to remove one suture of each knot, leaving only one stich. The benefit of this technic is to decrease the volume of material sutures below the acromion and the size and/or number of lateral anchorages dividing by two lateral sutures. Knot configuration, loop security and suture materials have been evaluated in previous biomechanical studies based on single-row constructs. The impact on pulling on the remaining stiches, like done in suture bridge, has not been previously evaluated. Static surgeon's knot with reversing half-hitches is associated 
with the best loop and knot security [5]. No study has measured the knot security relating to cutting distance from the knot or conserved suture limb (between the post and non-post limb) in mastress suturebridge. The purpose of this study was to define the more stable construct when a single limb is preserved in a suture bridge repair and evaluate the safe cutting distance from the knot. Additionally, we evaluate knot and loop security of this construct with five different types of suture material.

\section{Material And Methods}

This biomechanical study was performed following a custom made device based on a previous paper [5]. The evaluated construct is represented in the Figure 1. All knots were hand tied by a senior orthopedic surgery resident (C.T) on a custom-made support over two parallel metallic rods to create a $30-\mathrm{mm}$ suture loop, replicating the optimal loop security in arthroscopic cuff repair $[6,7]$. To recreate a standard arthroscopic procedure, knots were tied through an arthroscopic canula fixed to the support and using an arthroscopic knot pusher. This was done in a dry environment under direct visualization. A dynamometer (SF-100, Tanus $\AA$ ) was used to apply a constant load and realize the cyclic loading, up to 100 Newtons. For each experience, the traction was along a longitudinal axis between the knot and the dynamometer.

The static surgeon's knot was chosen for this study as it was the most commonly performed by the senior author (G.N). This knot comprised of a stack of 3 half-hitches (base knot) followed by three consecutives half-hitches on alternating posts (Figure 2).

We compared five different braided sutures and tapes: No. 2 Ethibond (Ethicon, Somerville, NJ), Hi-Fi (ConMed Linvatec, Largo, FL), Sutblue (SBM, Lourdes, France), SingleFlat (SBM, Lourdes, France), Hi-Fi Ribbon (ConMed Linvatec, Largo, FL). For each experiment, every single suture was used to tie the knot six times to be consistent with previous studies $[5,8]$.

The aim of the study was to know what the more stable condition of the knot is when cutting one stich of the suture before applying the second row.

Thus, we aimed to determine if there is a difference in knot loosening if we cut the post limb or the nonpost limb and at which distance from the knot should the suture be cut. This loosening is commonly defined as the sliding of the suture. If it does not occur, measurement of the length of the loop circumference is an objective way to compare construct loosening.

In the first experiment, a Surgeon's Knot was tied around the rods and a $10 \mathrm{~N}$ preload was applied on the post limb [9]. The non-post limb was cut at $1 \mathrm{~mm}(n=6)$ and $4 \mathrm{~mm}(n=6)$ with two distinct arthroscopic knot cutters (group A and B respectively). A tension load of $50 \mathrm{~N}$ was applied on the post limb for 10 seconds.

In the second experiment, we repeated the procedure with the post-limb cut at $1 \mathrm{~mm}(n=6)$ and $4 \mathrm{~mm}$ $(n=6)$ (group $\mathrm{C}$ and $\mathrm{D}$ respectively) and the tension load of $50 \mathrm{~N}$ applied on the non-post limb. 
Those two experiments allow us determining the limb to preserve and the safe cutting distance from the knot for each suture material tested.

Based on those results, in a third experiment, we tied the Surgeon's Knot around the rods, cut the determined limb at the more stable length from the knot and fixed the post limb with a vice after a $50 \mathrm{~N}$ pre-load). We applied an opposed traction of $100 \mathrm{~N}$ to search for mechanical failure [10] .

On the fourth experiment, we repeated the construct described above and performed a cyclic loading of $50 \mathrm{~N}$ during five seconds for 30 cycles to mimic chronic pulling strength as described in literature $[9,11]$.

For each experiment we took initial pictures, and videos during traction or cyclic loading to document mechanical failure. We reported loop circumference and knot slippage or breakage. We recorded the amount of suture elongation.

Statistical analysis was accomplished by use of R 3.5.0 software. Statistical significance was set for a $p$ value $<0.05$ and a power of 0.80 . Comparison of continuous variables was done using the Kruskall Wallis test.

\section{Results}

Experiments 1 and 2: Loop and knot security with different limb in traction and short limb length

Those two experiments evaluated the stability of different double-mastress suture-bridge constructs with a single limb preserved as following: a non-post limb cut at $1 \mathrm{~mm}$ distance in group A or $4 \mathrm{~mm}$ in group B; a post-limb cut at $1 \mathrm{~mm}$ distance in group $\mathrm{C}$ or $4 \mathrm{~mm}$ in group $\mathrm{D}$ (Figure 3 ).

Table 1 includes the loop circumferences of each group. The loop circumference of the static surgeon's knot tied with No. 2 Ethibond is, in all cases, the largest. There is a statistically significant difference between the tested braided sutures in all four groups for loop circumference and elongation $(p<0.01)$. Neither the preserved tensioned limb nor the cutting length altered the loop circumference when the groups were compared, independently of the suture type $(p=0.99)$. Suture elongation was minimal with Sutblue in all groups.

Table 1

Loop circumference in millimeters of static surgeon's knot tied with various sutures types after a $50 \mathrm{~N}$ tension load with different constructs (Group A: non-post limb cut at $1 \mathrm{~mm}$; Group B: non-post limb cut at $4 \mathrm{~mm}$; Group C: post limb cut at $1 \mathrm{~mm}$; Groupe D: non-post limb cut at $4 \mathrm{~mm}$ )

\begin{tabular}{|lllllll|}
\hline Group & Ethibond 2 & Hi-Fi & Sutblue & Hi-Fi Ribbon & SingleFlat 2 & p-value \\
\hline A & $33[33 ; 34.5]$ & $32[32 ; 32.75]$ & $30[30 ; 30.75]$ & $31[31 ; 32.5]$ & $30[29.25 ; 30]$ & $<0.01$ \\
\hline B & $33[33 ; 33]$ & $32[30.88 ; 32.75]$ & $31[30.25 ; 31]$ & $31[31 ; 31.75]$ & $30[29.25 ; 30]$ & $<0.01$ \\
\hline C & $33[33 ; 33]$ & $32.5[32 ; 33.75]$ & $30[30 ; 30]$ & $31[31 ; 31]$ & $29[28 ; 30]$ & $<0.01$ \\
\hline D & $33[33 ; 33]$ & $32.5[32 ; 33.75]$ & $30[30 ; 30]$ & $31[31 ; 31]$ & $29[28 ; 30]$ & $<0.01$ \\
\hline
\end{tabular}


Failure mechanism mainly occurred by knot slippage (Figure 3). It was the lowest in group $D$, when the non-post limb was tensioned and the post-limb was cut at $4 \mathrm{~mm}$, compared to the other groups (Table 2, $p<0.01$ ). All kind of suture slipped when the tensioned limb was the post-limb (group A and B). Knot breakage was observed only once with No. 2 Ethibond. There was no statistical difference between the sutures in group $\mathrm{A}, \mathrm{B}$ or $\mathrm{C}(p>0.05)$. In group $\mathrm{D}$, we noted knot slippage only with SingleFlat2 $(p=0.03)$.

Table 2

Failure mode in each tested construct according to suture type (Group A: non-post limb cut at $1 \mathrm{~mm}$; Group B: non-post limb cut at $4 \mathrm{~mm}$; Group C: post limb cut at $1 \mathrm{~mm}$; Group D: non-post limb cut at 4 $\mathrm{mm})$

\begin{tabular}{|lllll|}
\hline & Group A & Group B & Group C & Group D \\
\hline No 2. Ethibond & 83\% Slipped, $n=5$ & $\begin{array}{l}50 \% \text { Slipped, } n=3 \\
17 \% \text { Broke, } n=1\end{array}$ & $17 \%$ Slipped, $n=1$ & 0 \\
\hline Hi-Fi & 83\% Slipped, $n=5$ & $100 \%$ Slipped, $n=6$ & 0 & 0 \\
\hline Sutblue & $67 \%$ Slipped, $n=4$ & $67 \%$ Slipped, $n=4$ & $17 \%$ Slipped, $n=1$ & 0 \\
\hline Hi-àlFi Ribbon & 100\% Slipped, $n=6$ & $83 \%$ Slipped, $n=5$ & $17 \%$ Slipped, $n=1$ & 0 \\
\hline SingleFlat 2 & $83 \%$ Slipped, $n=5$ & $67 \%$ Slipped, $n=4$ & $33 \%$ Slipped, $n=2$ & $50 \%$ Slipped, $n=3$ \\
\hline
\end{tabular}

\section{Experiment 3: Loop and knot security after a 100-N single- pull load}

The loop circumference was significantly higher for all sutures after a 100-N single pull load on a suture bridge construct $(p<0.01)$. There was a significant amount of elongation for all the tested sutures $(p<0.0001)$. Elongation of the loop was minimal with Sutblue $(0,05 \pm 0,028)$ followed by Hi-Fi $(0,1 \pm 0,04)$, SingleFlat2 $(0,1 \pm 0,02)$ and No. 2 Ethibond $(0,22 \pm 0,03)$. It was the largest with Hi-Fi Ribbon $(0,23 \pm 0,03)$.

Failure occurred only by knot slippage and only with tapes (Table 3 ). We found no significant difference between the suture type $(p=0,47)$. Knot slippage was observed during the pre-tensioned step at $50-\mathrm{N}$ when the post limb was fixed with the vice. 
Table 3

Failure mode after a 100-N single-pull load

\begin{tabular}{|llll|}
\hline Suture & $\boldsymbol{n}$ & Knot slippage & Knot breakage \\
\hline Ethibond 2 & 6 & 0 & 0 \\
\hline Hi-Fi & 6 & 0 & 0 \\
\hline Sutblue & 6 & 0 & 0 \\
\hline Hi-Fi Ribbon & 6 & $17 \%, n=1$ & 0 \\
\hline SingleFlat 2 & 6 & $33 \%, n=2$ & 0 \\
\hline
\end{tabular}

\section{Experiment 4: Loop and knot security after cyclic loading}

Loop circumference and elongation were significantly higher for all sutures after cyclic loading $(p<0.01)$. Elongation of the loop was minimal with Sutblue $(0,03 \pm 0,02)$ followed by Hi-Fi $(0,09 \pm 0,05)$, SingleFlat2 $(0,14 \pm 0,01)$ and No. 2 Ethibond $(0,15 \pm 0,03)$ It was the largest with Hi-Fi Ribbon $(0,17 \pm 0,04)$.

No knot breakage was observed. Knot slippage concerned six out of the thirty knots tested. We found a significant difference between the suture types, and tapes were the less secure material $(p=0.043$, Table 4).

Table 4

Failure mode after cyclic loading

\begin{tabular}{|llll|}
\hline Suture & $\boldsymbol{n}$ & Knot slippage & Knot breakage \\
\hline Ethibond 2 & 6 & $17 \%, n=1$ & 0 \\
Hi-Fi & 6 & 0 & 0 \\
\hline Sutblue & 6 & 0 & 0 \\
\hline Hi-Fi Ribbon & 6 & $17 \%, n=1$ & 0 \\
\hline SingleFlat 2 & 6 & $67 \%, n=4$ & 0 \\
\hline
\end{tabular}

\section{Discussion}

To our knowledge, this study is the first to evaluate the mechanical properties of this specific suturebridge construct and the safety of the cutting distance from the knot. A suture bridge construct with the non-post limb preserved and the post-limb cut at a 4-mm distance from the knot provides with the best security. Tapes tested in our study should not be recommended for this type of construct.

Healing of the cuff is the main objective of shoulder cuff repair. Failed repair are correlated with poor outcomes and risk of progressive osteoarthritis. Several causes of failure are well identified; among them early failure or loosening of the suture material should be correlated to non-healing $[12,13]$. Suture bridge 
have been developed to stabilize the first row of fixation and increase the contact between the bone of the tendon. Clinical results confirm its benefit but increasing the number of lateral stiches increase the volume of material in the subacromial space and the size and/or number of anchors used for the lateral row. Removing one suture can decrease those risks but should not compromise stability (Figure 4).

Numerous publications have focused on knot and loop security in a single-row construct with different types of knot tested. Our results are consistent with previous studies that demonstrate the best security with static surgeon's knot and the importance of reversed half-hitches to secure the knot $[5,14]$. They highlighted that the construct itself matters in knot security in a double-row configuration. When tension was applied on the post limb, it was associated with elongation and slippage of the last half-hitches. On the contrary, applying tension on the non-post limb allowed for a stable construct with less knot failure. It is also safer to cut the knot at a 4-mm distance than 1-mm distance.

To ensure the best loop security, we customized a stable mechanical model where the rods were separated to create a $30-\mathrm{mm}$ suture loop as reported in literature [7]. The preserved limb and the cutting distance from the knot did not interfere with loop elongation $(p=0.99)$. Loop circumference increased with single-pull load and cyclic loading for all kind of sutures tested $(p<0.01)$.

We found that knot failure was limited when the non-post limb is preserved, and the post-limb cut at a 4$\mathrm{mm}$ distance from the knot. It occurred mainly with tapes and by slippage. The results are similar whether it is a single-pull load or a cyclic load experiment. Previous studies have demonstrated the advantages of using tapes rather than sutures in rotator cuff repair: higher load-to-failure, higher stiffness, and higher contact pressure between the bone and the tendon $[15,16]$. Despite their biomechanical properties, Liu \& al. observed that tapes do not influence the retear rate [17]. Additionally, tapes have not been evaluated considering knot security. Considering the existing literature and our results, we do not recommend using tapes for double-row configuration constructs involving knots tying.

The strengths of this study are that the knots were all tied by a single surgeon, with the use of arthroscopic instrument and a standardized loop size, under same load device, following previous validated protocols [5-7].

The limitations are the variation in the tension applied to each knot as it was done by a surgeon and not mechanically by a machine. Even if the load applied was consequent, we could not study the load-tofailure with the dynamometer at our disposition. Experiments were done in a dry environment and our results might differ when sutures are soaked in a biological media reproducing human body conditions. Rousseau \& al. have shown that body fluid immersion influence knot slippage and differences exists between UHMWPE sutures [10]. This study confirmed that UHMWPE sutures were safer than No 2. Ethibond and tapes in this construct. Statistical difference between suture material is not available as it would have come from multiple tests with no statistical power.

Additional studies could provide with knot and loop security in a wet environment and help differentiate more precisely sutures and tapes in this suture-bridge construct. 


\section{Conclusion}

A suture-bridge construct with the non-post limb preserved and the post-limb cut at a 4-mm distance from the knot provides with the best security. Sutures we tested in our biomechanical study are safer than tapes in this double-row suture bridge single stich configuration.

\section{Declarations}

\section{Funding:}

No financing was received for this study

\section{Competing interest:}

The authors have no relevant financial or non-financial interests to disclose.

\section{Ethical approval:}

This is a biomechanical study that was not done on human or animal subjects. Thus, it does not require an ethical approval.

\section{Consent to participate:}

This is a biomechanical study that was not done on human or animal subjects. Thus, it does not require a consent to participate.

\section{Consent to publish:}

This is a biomechanical study that was not done on human or animal subjects. Thus, it does not require a consent to publish.

Availability of data and material:

This is a biomechanical study that was not done on human or animal subjects. All the data and material can be transferred if needed.

\section{Aknowledgments:}

We would like to thank the companies ConMed (Linvatec, Largo, FL), and SBM (Lourdes, France) for providing us with the sutures.

\section{Authors' contributions:}

S Abihssira helped with the experiments and wrote the manuscript.

C Thirache participated in the design of the study and performed all the experiments. 
C Camas provided with the illustrations of the manuscript

P-A Bouché performed all the statistical analysis.

G Nourissat designed the study and revised the manuscript.

All authors read and approved the final manuscript.

\section{References}

1. Gartsman GM, Drake G, Edwards TB, Elkousy HA, Hammerman SM, O'Connor DP, et al. (2013) Ultrasound evaluation of arthroscopic full-thickness supraspinatus rotator cuff repair: single-row versus double-row suture bridge (transosseous equivalent) fixation. Results of a prospective, randomized study. J Shoulder Elbow Surg;22:1480-7. https://doi.org/10.1016/j.jse.2013.06.020.

2. Hein J, Reilly JM, Chae J, Maerz T, Anderson K. (2015) Retear Rates After Arthroscopic Single-Row, Double-Row, and Suture Bridge Rotator Cuff Repair at a Minimum of 1 Year of Imaging Follow-up: A Systematic Review. Arthrosc J Arthrosc Relat Surg ;31:2274-81.

https://doi.org/10.1016/j.arthro.2015.06.004.

3. Mall NA, Lee AS, Chahal J, Van Thiel GS, Romeo AA, Verma NN, et al. (2013) Transosseous-Equivalent Rotator Cuff Repair: A Systematic Review on the Biomechanical Importance of Tying the Medial Row. Arthrosc J Arthrosc Relat Surg;29:377-86. https://doi.org/10.1016/j.arthro.2012.11.008.

4. Anderl W, Heuberer PR, Laky B, Kriegleder B, Reihsner R, Eberhardsteiner J. (2012) Superiority of bridging techniques with medial fixation on initial strength. Knee Surg Sports Traumatol Arthrosc;20:2559-66. https://doi.org/10.1007/s00167-012-1922-9.

5. Lo IKY, Burkhart SS, Chan KC, Athanasiou K. (2004) Arthroscopic knots: Determining the optimal balance of loop security and knot security. Arthrosc J Arthrosc Relat Surg;20:489-502. https://doi.org/10.1016/j.arthro.2004.03.005.

6. Lo IKY, Ochoa E, Burkhart SS. (2010) A Comparison of Knot Security and Loop Security in Arthroscopic Knots Tied With Newer High-Strength Suture Materials. Arthrosc J Arthrosc Relat Surg;26:S120-6. https://doi.org/10.1016/j.arthro.2009.12.009.

7. Ergün S, Akgün U, Karahan M. (2020) The effect of loop size on loop security and elongation of a knot. Orthop Traumatol Surg Res;106:35-8. https://doi.org/10.1016/j.otsr.2019.10.008.

8. Meyer DC, Bachmann E, Lädermann A, Lajtai G, Jentzsch T. (2018) The best knot and suture configurations for high-strength suture material. An in vitro biomechanical study. Orthop Traumatol Surg Res;104:1277-82. https://doi.org/10.1016/j.otsr.2018.08.010. 
9. Barber FA, Herbert MA, Beavis RC. (2009) Cyclic Load and Failure Behavior of Arthroscopic Knots and High Strength Sutures. Arthrosc J Arthrosc Relat Surg;25:192-9.

https://doi.org/10.1016/j.arthro.2008.09.010.

10. Rousseau B, Diop A, Atlan F, Priam S, Aim F, Nourissat G, et al. (2013) Influence of prolonged immersion on the resistance of arthroscopy knots in biological media. Orthop Traumatol Surg Res;99:138-44. https://doi.org/10.1016/j.otsr.2012.09.020.

11. Busfield BT, Glousman RE, McGarry MH, Tibone JE, Lee TQ. (2008) A Biomechanical Comparison of 2 Technical Variations of Double-Row Rotator Cuff Fixation: The Importance of Medial Row Knots. Am J Sports Med;36:901-6. https://doi.org/10.1177/0363546507312640.

12. Duquin TR, Buyea C, Bisson LJ. (2010) Which Method of Rotator Cuff Repair Leads to the Highest Rate of Structural Healing?: A Systematic Review. Am J Sports Med;38:835-41. https://doi.org/10.1177/0363546509359679.

13. Cummins CA, Murrell GAC. (2003) Mode of failure for rotator cuff repair with suture anchors identified at revision surgery. J Shoulder Elbow Surg;12:128-33. https://doi.org/10.1067/mse.2003.21.

14. Elkousy HA, Sekiya JK, Stabile KJ, McMahon PJ. (2005) A biomechanical comparison of arthroscopic sliding and sliding-locking knots. Arthrosc J Arthrosc Relat Surg;21:204-10. https://doi.org/10.1016/j.arthro.2004.09.024.

15. Boksh K, Haque A, Sharma A, Divall P, Singh H. (2021) Use of Suture Tapes Versus Conventional Sutures for Arthroscopic Rotator Cuff Repairs: A Systematic Review and Meta-analysis. Am J Sports Med https://doi.org/10.1177/0363546521998318.

16. Borbas P, Fischer L, Ernstbrunner L, Hoch A, Bachmann E, Bouaicha S, et al. (2021) High-Strength Suture Tapes Are Biomechanically Stronger Than High-Strength Sutures Used in Rotator Cuff Repair. Arthrosc Sports Med Rehabil;3:e873-80. https://doi.org/10.1016/j.asmr.2021.01.029.

17. Liu RW, Lam PH, Shepherd HM, Murrell GAC. (2017) Tape Versus Suture in Arthroscopic Rotator Cuff Repair: Biomechanical Analysis and Assessment of Failure Rates at 6 Months. Orthop J Sports Med https://doi.org/10.1177/2325967117701212.

\section{Figures}




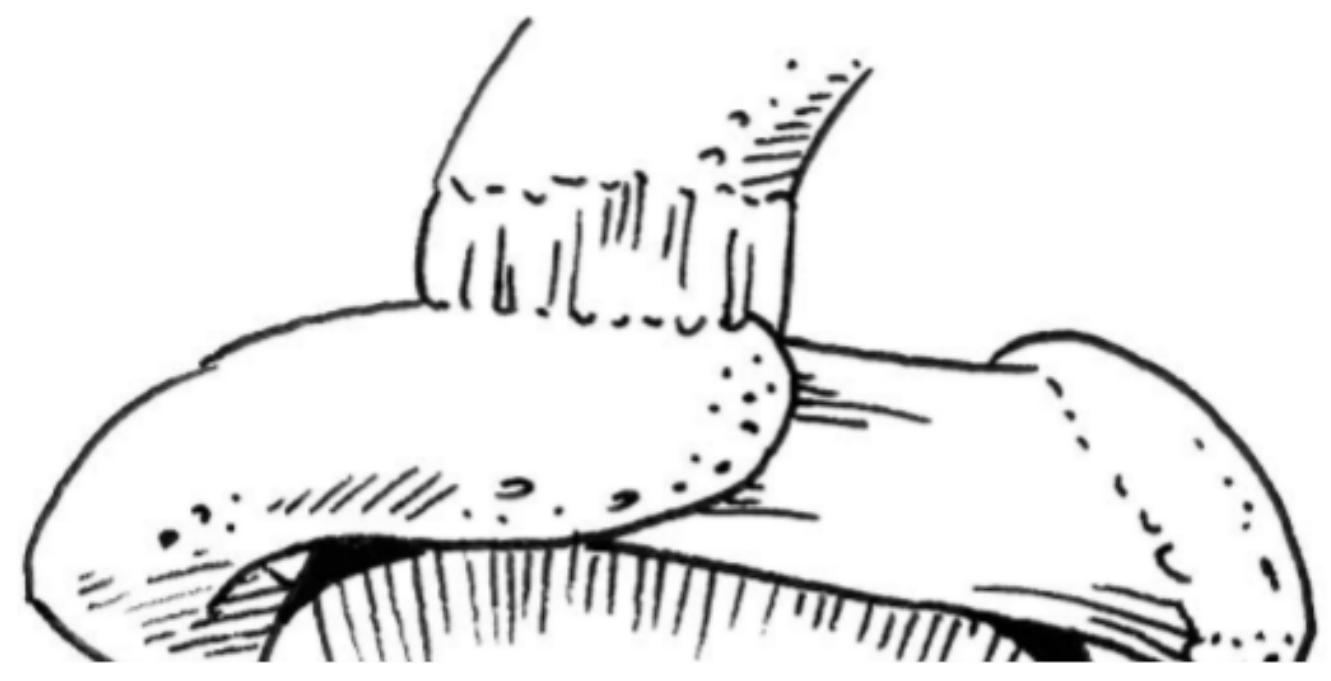

Figure 1

Views of the suture-bridge configuration tested 


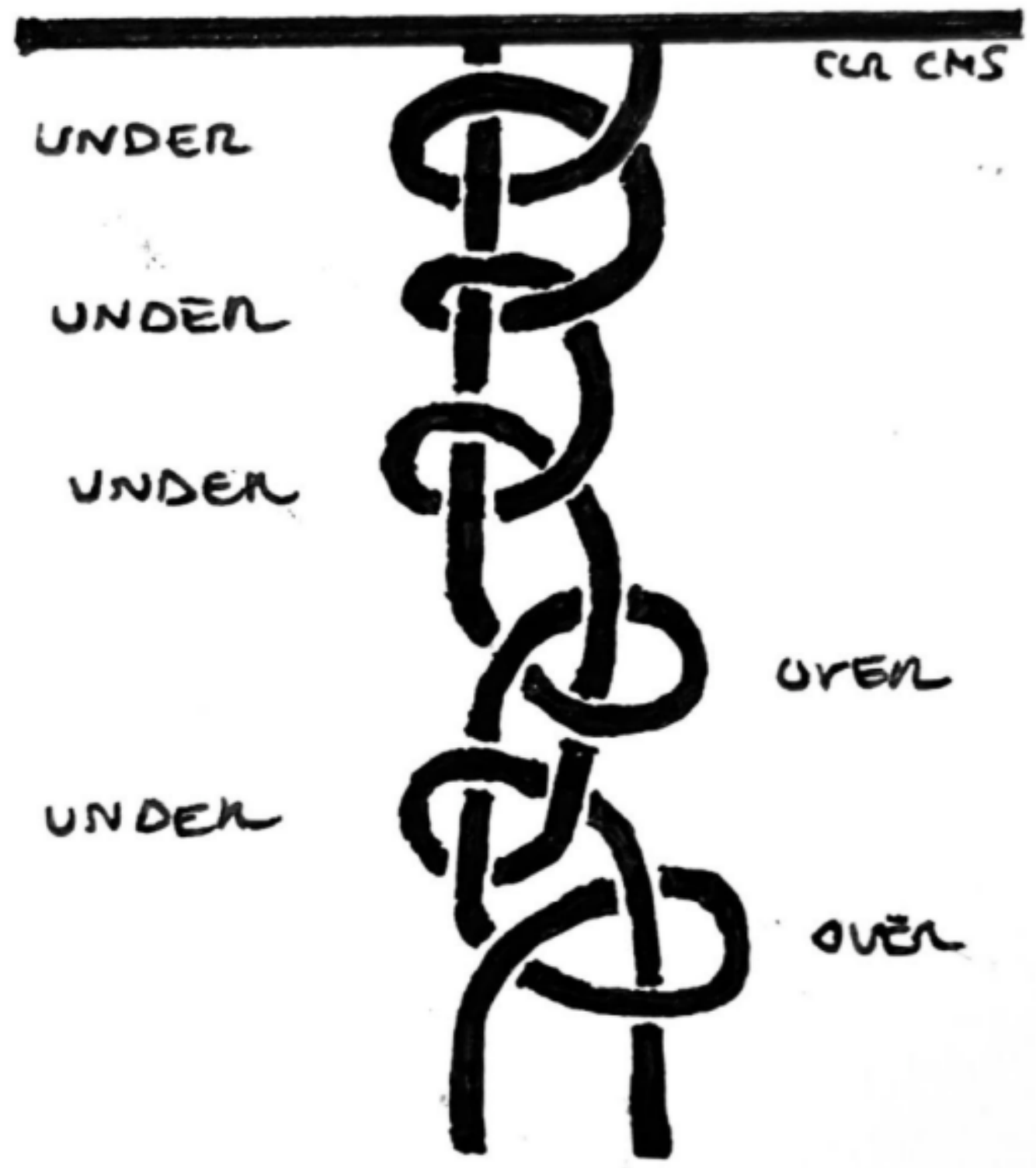

Figure 2

Static surgeon's knot configuration

Figure 3

a. Aspect of a Static surgeon's knot before tension with tape (SingleFlat ${ }^{\circledR}$ ) b. Aspect of a stable construct in group D c. Aspect of knot slippage in group $A($ knots tightened with Sutblue $\AA$ ) The red arrow indicates the post-limb in each case. 


\section{Figure 4}

Suture-bridge equivalent construct a. First row tightened with a Static surgeon's knot using a Hi-Fi suture before applying the second row, b. Final construct of suture bridge with single lateral stich configuration 